\title{
Prognosis for rubeosis iridis following central retinal vein occlusion
}

\author{
STEPHEN H. SINCLAIR AND EVANGELOS S. GRAGOUDAS \\ From the Eye Research Institute of Retina Foundation, and the Retina Service, \\ Massachusetts Eye and Ear Infirmary, Boston, Massachusetts
}

SUMMARY The records of 57 patients with recent central retinal vein occlusion were reviewed in order to predict the development of rubeosis iridis (RI) and neovascular glaucoma (NVG) from the initial clinical examination, colour fundus photographs, and fluorescein angiograms. Twelve patients $(21 \%)$ developed both RI and NVG, and this complication appeared to be correlated most significantly with clinical and fluoroangiographic evidence of severe retinal ischaemia. The correlation between RI and other findings was as follows: widespread capillary occlusion ( $86 \%$ developed RI); absent perifoveal network ( $80 \%)$; 10 or more cotton-wool spots (75\%); A-V transit time of greater than 20 seconds (75\%); severe large vessel leakage (31\%); and severe retinal oedema $(60 \%)$. Less significant correlation of RI was obtained with poor visual acuity and with associated systemic diseases. Factors of no statistical prognostic value included the patient's age, history, or evidence of pre-existing open-angle glaucoma, degree of fundus haemorrhage, capillary dilatation, disc oedema, disc capillary dilatation and leakage, leakage of small vessels in the posterior pole, and development of disc collateral vessels. Multivariate linear discriminant analysis of the data supported the above factors as being the variables that best predicted the development of RI, and a derived linear equation predicted $91 \%$ of all patients studied who had central retinal vein occlusion and who developed rubeosis iridis.

Rubeosis iridis (Rl) and neovascular glaucoma (NVG) are severe complications of central retinal vein occlusion (CRVO) which occur in $12 \%$ to $30 \%$ of all cases (Smith, 1961; Vannas, 1961; Laatikainen and Kohner, 1976). The stimulus for anterior segment neovascularisation is poorly understood but is thought to be related to severe retinal ischaemia (Smith, 1961; Ashton, 1961; Shilling and Kohner, 1976). Fluorescein angiography adds considerable information to the understanding of the pathophysiology of CRVO by enabling qualitative evaluation of the retinal vascular responses to an ischaemic lesion. These responses include retinal capillary nonperfusion, dilatation of small vessels, microaneurysm formation, and abnormal vascular permeability secondary to endothelial cell damage. Studies in diabetics (Laatikainen and Kohner, 1976; Kohner et al., 1976) have suggested a relation between the development of fundus or iris neovascularisation and widespread areas of retinal capillary nonperfusion. A similar relation has been reported for branch vein occlusion (Kohner et al., 1976; Sedney, 1976; Laatikainen and Blach, 1977) and CRVO (Laatikainen and Kohner, 1976; Hayreh, 1976, Kottow et al., 1976; Laatikainen, 1977a).

Prognosticating this complication has become important, since recent studies (Sedney, 1976; May et al., 1976; Laatikainen, 1977b) have reported that panretinal photocoagulation may be used to reverse or prevent iris neovascularisation after CRVO. It has been suggested, however, that treatment should be completed either early in the course of rubeosis, before extensive peripheral anterior synechiae are established, or prophylactically before clinical rubeosis is recognised. This obviously necessitates the early identification of those patients with CRVO who are at high risk of developing neovascular complications. This study was undertaken to extend previous studies (Laatikainen and Kohner, 1976; Laatikainen and Blach, 1977) in order to determine the features of either the initial clinical examination, the fluorescein 
angiogram, or both, that might be statistically correlated with the subsequent development of anterior segment neovascularisation.

\section{Materials and methods}

The records of patients with CRVO who had been referred to the Massachusetts Eye and Ear Infirmary Fluorescein Service were reviewed for development of clinically unequivocal rubeosis iridis, neovascular glaucoma, or both. Only patients with relatively 'fresh' vein occlusions were selected; patients with 'old' occlusions, either by history or by fundus criteria, were excluded. The duration of symptoms prior to the initial examination of the 57 patients included is shown in Table 1. Fifty-four cases were unilateral and 3 bilateral, in which a CRVO had occurred recently after an older occlusion in the fellow eye. The average age of the patients was 60 years, with a range of 23 to 93 years. All patients were followed up until resolution of the CRVO or until they developed clinically unequivocal rubeosis iridis or neovascular glaucoma. The average follow-up period of eyes that did not develop rubeosis was 10 months (range 4 to 36 months). Most patients received no treatment after initial evaluation; only a few were treated with antifibrinolytic agents, anticoagulants, or steroids.

Patients' records, which included stereo fundus photographs, stereo fundus fluorescein angiograms, and data from the initial and follow-up examinations, were reviewed for the following details: (1) history: duration and type of symptoms, age, associated systemic, ocular, or orbital disease; (2) initial ocular examination: visual acuity, intraocular pressure, afferent pupillary defect; fellow eye visual acuity, intraocular pressure, disc or field changes, and retinal pathology; (3) course: final visual acuity, time required for resolution of haemorrhages and oedema, development of macular cysts, disc collaterals, unequivocal iris neovascularisation (aberrant surface vessels visible on biomicroscopy), or neovascular glaucoma.

Table 1 Duration of symptoms in patients with CRVO

\begin{tabular}{ll}
\hline Duration of symptoms & No. of patients $(\%)$ \\
\hline $1 \mathrm{mo}$ & $29(51)$ \\
$2-3 \mathrm{mo}$ & $13(23)$ \\
$>3 \mathrm{mo}$ & $6(11)$ \\
Undetermined length of time & $5(9)$ \\
Routine examination without symptoms & $4(7)$ \\
\hline
\end{tabular}

CRVO $=$ central retinal vein occlusion.
For the purpose of this study the photographed retina of the involved eye was divided into 3 territories for evaluation of various areas: (1) macula: area within a 2-disc radius from the fovea; (2) posterior pole: disc and area within the major temporal vascular arcades, except for the macular area described above; (3) periphery: area peripheral to the disc and major temporal arcades. Colour stereo fundus photographs were used to assess the severity or extent of retinal haemorrhages, oedema, and cotton-wool spots within these 3 territories, and collateral disc vessels.

In each of the above territories the intraretinal, deep, superficial, and subhyaloid retinal haemorrhages were graded: none ( 0 ), mild (1), moderate (2), or severe (3) (the number in parentheses is the grade of severity for each variable that we included in the multivariate discriminant analysis; see 'Statistical analysis'). Retinal oedema was graded: none (0), mild (1), or severe (2), with notation of the formation of macular cysts (3). The number of cottonwool spots was recorded, as well as their papillary, peripapillary, or peripheral location. Opticociliary veins were either present (1) or absent (0).

Stereo fluorescein angiograms were used to evaluate retinal capillary nonperfusion, capillary dilatation, and vascular permeability. In each of the 3 territories we evaluated the integrity of the retinal capillary bed during early dye transit as well as could be determined through and between the retinal haemorrhages. Even in cases with extensive haemorrhages ischaemic areas could be delineated with relative ease (Fig. 1). In the macular area, the perifoveal capillary arcade was described as present (0), broken (1), or absent (2) (no perfused capillaries within a one-disc radius of the fovea). In the posterior pole and periphery the total area of capillary nonperfusion was graded from no capillary occlusion (0) to scattered foci of occluded capillaries totalling less than 1 quadrant (1), 1 to 2 quadrants (2), or severe, contiguous occlusion totalling 3 to 4 quadrants (3). In each territory of the angiogram the total area of capillary dilatation or microaneurysm formation was estimated and graded: none ( 0$)$, scattered foci totalling less than 1 quadrant (1), 1 to 2 quadrants (2), or 3 to 4 quadrants (3). Ischaemic damage to the vascular endothelium was determined by the degree of abnormal permeability observed in the small vessels (capillaries and venules) or the large veins in 2 retinal territoriesthe posterior retina (macula and posterior pole) and the peripheral retina. Permeability was graded: none (0), mild staining (1), mild leakage (2), or severe leakage (3). Forty fluorescein transits were timed, and the arteriovenous transit time was estimated, from the appearance of dye in the 

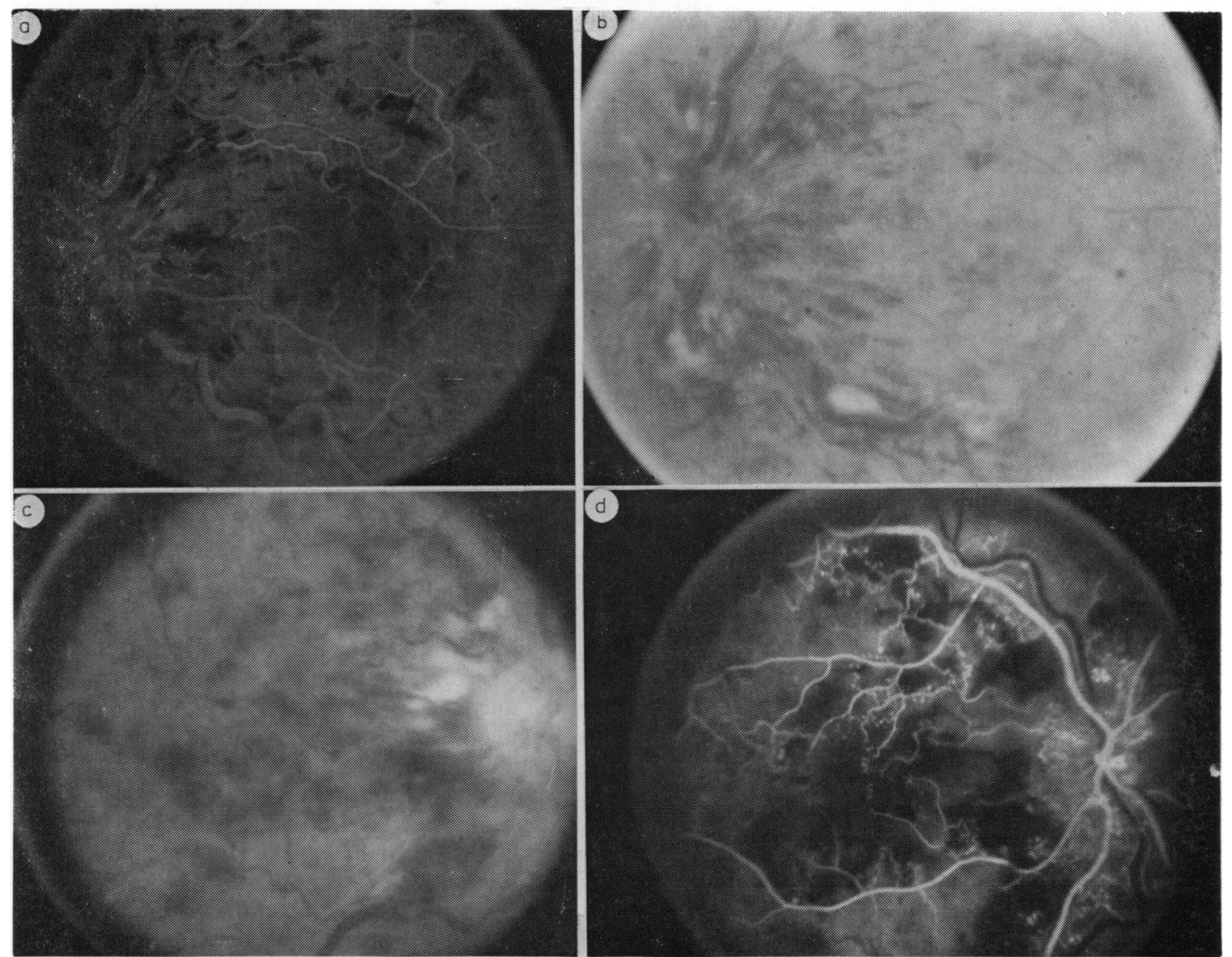

Fig. 1 Fundus photograph and mid-capillary-transit fluorescein angiogram of patient with CRVO. A, B: Mild, scattered foci of capillary occlusion; C, D: Extensive capillary occlusion

retinal arteries to the complete filling of the major central retinal veins.

\section{STATISTICAL ANALYSIS}

All of the collected data and their association with rubeosis iridis were subjected to statistical analysis by $\chi^{2}$ tests for the resultant $3 \times 2$ or $4 \times 2$ tables. The data were also subjected to a computer analysis which derived a multivariate, linear, discriminant coefficient equation. This analysis evaluates the potential of each variable, alone and in combination with others, to predict which patients will develop rubeosis iridis as a complication of CRVO. The programme derives a linear equation of those variables that are the best predictors. A weighted coefficient, assigned to each variable, is multiplied by the graded severity of the variable: $\mathbf{X}=\mathbf{K}+$ $C_{1}\left(V_{1}\right)+C_{2}\left(V_{2}\right)+C_{3}\left(V_{3}\right)+\ldots+C_{n}\left(V_{n}\right)$ where $K$ is a constant, $C_{1} \ldots C_{n}$ are the weighted coefficients for the best-predicting variables, and $V_{1} \ldots V_{n}$ are the graded values of the patient for each variable. The resultant discriminant score (X) indicates the tendency of that patient to develop rubeosis iridis. We can thus derive a weighted combination of the best-predicting variables which may serve better than any 1 variable alone to predict which patients will develop rubeosis iridis. A discriminant score was calculated for each patient by means of coefficients of the best-predicting variables derived by the programme along with their graded values (the parenthetical values described above).

\section{Results}

Rubeosis iridis developed in 12 of 57 patients ( $21 \%$ ) with early CRVO within a period of 3 weeks to 7 months after the onset of symptoms (Table 2), 


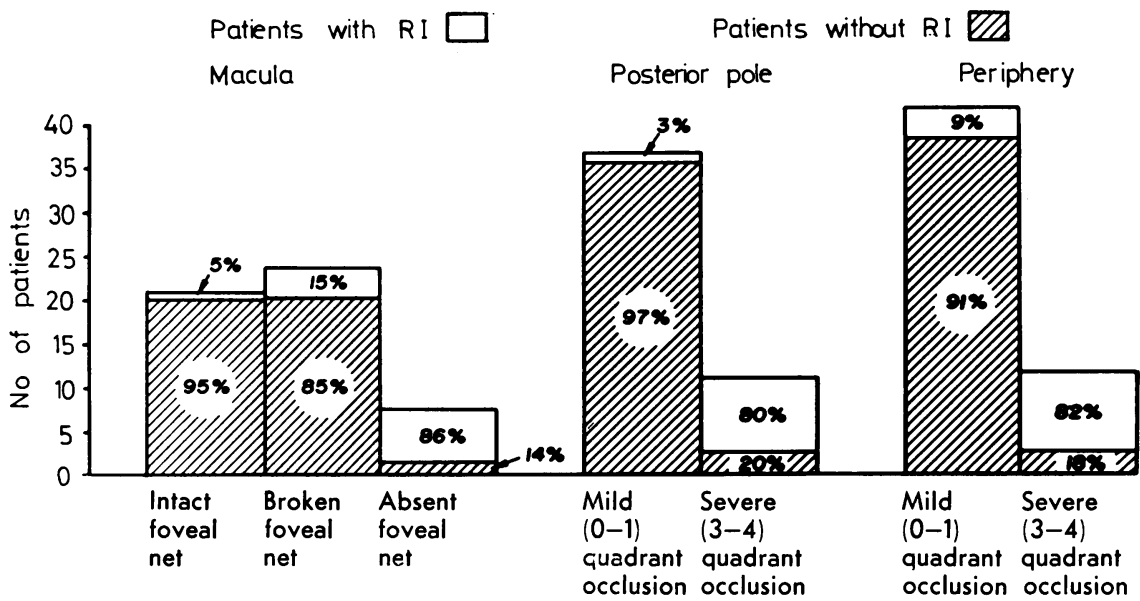

Table 2 Interval between onset of symptoms and development of rubeosis in CRVO

\begin{tabular}{ll}
\hline Interval (months) & No. of patients \\
\hline$<1$ & 1 \\
$1-$ & 2 \\
$2-$ & 3 \\
$3-$ & 3 \\
$4+$ & 3 \\
Total & 12 \\
\hline
\end{tabular}

CRVO $=$ central retinal vein occlusion.

and in all these patients angle neovascularisation and glaucoma ensued. The development of rubeosis iridis was most significantly correlated with those features of the initial clinical examination and fluorescein angiogram that indicated severe retinal ischaemia. As shown in Fig. 2, rubeosis iridis correlated most directly with the extent of capillary nonperfusion that was observed on the fluorescein angiogram. Rubeosis developed in $80-86 \%$ of eyes with an absent parafoveal net of 3 to 4 quadrants of posterior pole or peripheral capillary occlusion, but appeared in only 3-9\% of those with an intact parafoveal net and less than 1 quadrant of capillary occlusion. These results were significant, $P<0.001$ level. The view of the perifoveal arcade was sufficiently blocked by haemorrhage to prevent evaluation in 6 patients.

Less significant correlations were obtained for other indices of retinal ischaemia. Among patients with greater than 10 cotton-wool spots (CWS) rubeosis developed in $75 \%$ (Fig. 3), whereas the incidence was $24 \%$ in patients in whom less than 10 CWS were observed, and only $14 \%$ if no CWS were noted $(P<0.001)$. Increased vascular permeability (Fig. 4) and retinal oedema (Fig. 5) were even less well correlated. Among patients with severe grades of leakage and oedema rubeosis iridis and neovascular glaucoma developed in $31-60 \%$, but the incidence decreased to $4-10 \%$ in those with no leakage or mild staining of the vessel walls $(\mathrm{P}<0.05)$. A prolonged A-V transit of more than 20 seconds was associated with a significantly higher incidence of rubeosis iridis, $75 \%$, whereas the condition developed in only $10 \%$ of those with a transit of less than 20 seconds $(P<0.001$; Fig. 6$)$.

Although a significant correlation between the initial visual acuity and subsequent anterior segment neovascularisation was noted, this relationship was less marked than the previous indices. Rubeosis developed in $36 \%$ of the patients whose initial acuity was $6 / 60(20 / 200)$ or worse, but arose in only $7 \%$ if the acuity was $6 / 30(20 / 100)$ or better (P $<0.01)$.

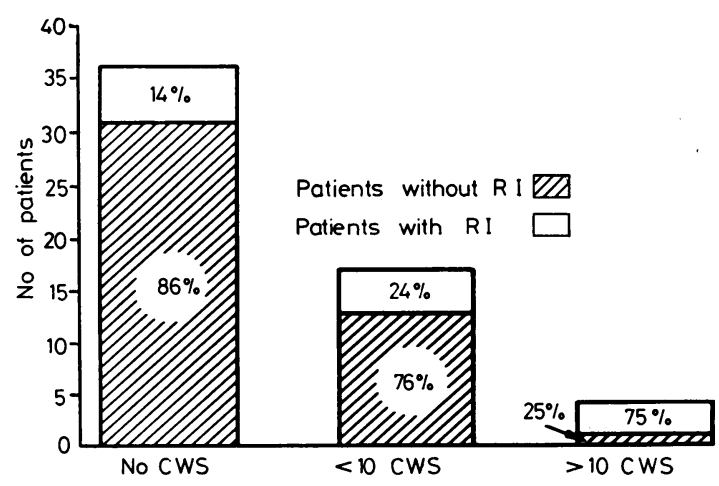

Fig. 3 Correlation of rubeosis iridis $(R I)$ with number of fundus cotton-wool spots (CWS) following $C R V O$ 
Patients with RI $\square$ Patients without RI $\mathbb{Z}$ Posterior pole Periphery
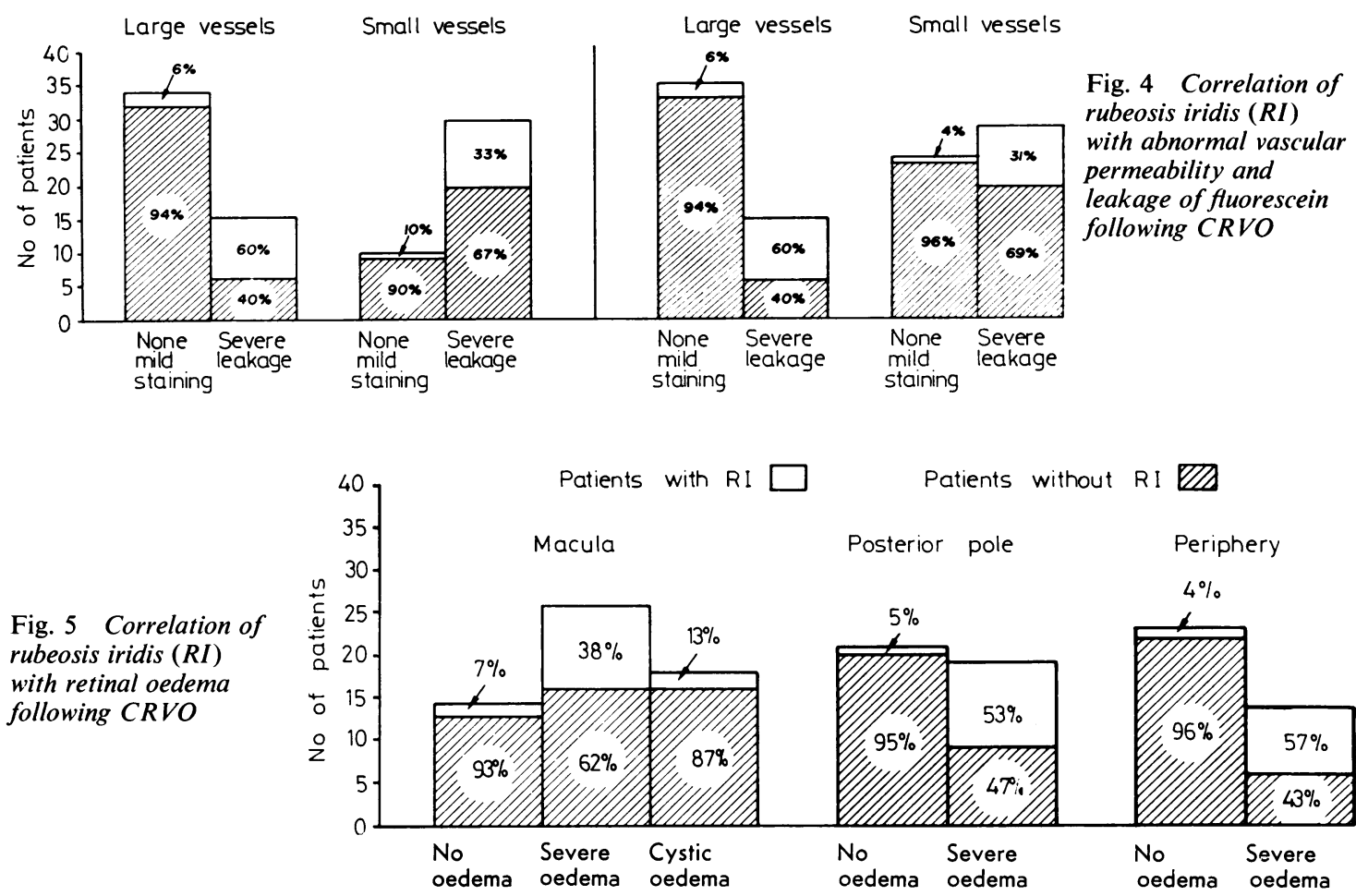

Moderate or severe degrees of arterial, venous, or diabetic fellow-eye fundus abnormalities were not correlated with a statistically different prognosis $(24 \%, 33 \%$, and $20 \%$ developed RI respectively) than a normal fundus in the fellow eye $(17 \%$ developed RI). Other factors which were studied but found to be of no statistical prognostic value included the following: the extent of fundus haemorrhage in the involved eye, the extent of capillary dilatation, the degree of disc oedema, disc capillary dilatation or leakage, leakage of small vessels in the posterior pole, and the presence of established disc collateral vessels.

Age was not correlated with neovascularisation; the average age of those with or without rubeosis was the same $(63 \pm 19 \mathrm{yr}$ versus $59 \pm 16 \mathrm{yr}$ respectively). Furthermore, when patients were divided into young $(\leqslant 45 \mathrm{yr})$ and old $(>45)$ age groups, there was no significant difference in the percentage of each group of patients $(18 \%$ and $22 \%)$ in whom rubeosis developed.

A relatively small number of patients with rubeosis had associated systemic disease (Table 3), which included diabetes, hypertension, arthritis, arteritis, atherosclerotic cardiac and/or peripheral vascular disease, and hypercoagulable syndromes (including minor platelet dysfunctions and polycythaemia). Six patients had open-angle glaucoma (with a history of glaucoma or evidence of raised intraocular pressure (IOP) with pathological cupping in the fellow eye), and 6 patients had ocular

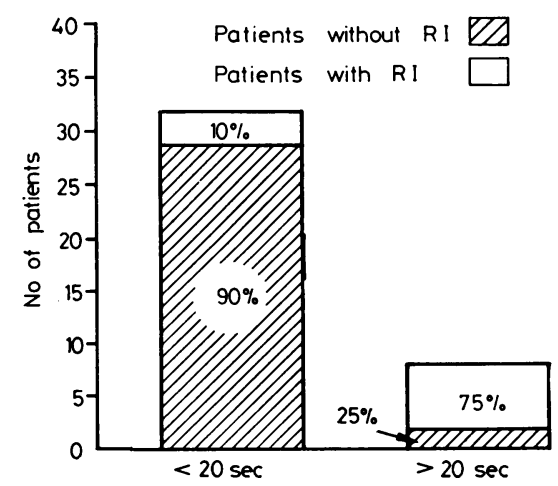

Fig. 6 Correlation of rubeosis iridis $(R I)$ with fluorescein $A-V$ transit time following CRVO 
Table 3 Associated diseases in patients with CRVO and rubeosis iridis

\begin{tabular}{lll}
\hline Disease & $\begin{array}{l}\text { No. of patients } \\
\text { with CRVO }\end{array}$ & $\begin{array}{l}\text { No. of patients } \\
\text { with RI }\end{array}$ \\
\hline None & 13 & $1(8 \%)$ \\
Hypertension & 34 & $7(21 \%)$ \\
Arthritis or arteritis & 4 & $1(25 \%)$ \\
$\begin{array}{l}\text { Azotaemia } \\
\begin{array}{l}\text { Atherosclerotic cardiac/peripheral } \\
\text { vascular disease }\end{array}\end{array}$ & 4 & $0(0 \%)$ \\
$\begin{array}{l}\text { Hypercoagulable/hyperviscosity } \\
\text { syndrome }\end{array}$ & 4 & $1(14 \%)$ \\
$\begin{array}{l}\text { Diabetes } \\
\text { Primary open-angle glaucoma }\end{array}$ & 6 & $3(75 \%)$ \\
Ocular hypertension & 9 & $2(22 \%)$ \\
\hline
\end{tabular}

$\mathrm{CRVO}=$ central retinal vein occlusion. $\mathrm{RI}=$ rubeosis iridis.

hypertension (IOP $>22 \mathrm{mmHg}$ without pathological disc cupping). Taken individually, none of these conditions was statistically significant when compared with the incidence of rubeosis in the absence of systemic or ocular disease. Grouped together, however, the incidence of rubeosis occurring with any systemic or ocular disease $(25 \%)$ was significant when compared with the incidence of rubeosis with CRVO without any associated systemic disease $(8 \%)(P<0.01)$.

The condition of the perifoveal network and the observation of macular oedema on the initial fluorescein angiogram correlated well with the initial visual acuity as well as the prognosis for late acuity. This supports findings of previous studies (Laatikainen and Kohner, 1976; Kohner, 1976). If on the initial fluorescein angiogram the perifoveal capillary arcade was judged intact, then the initial acuity was related to the degree of cystic maculopathy. Patients with mild leakage achieved 6/12 $(20 / 40)$ or better, whereas those with severe oedema, cysts, or both had 6/7.5 (20/50) to 6/60 (20/200) vision. If the perifoveal capillary arcade was broken or absent, the leakage and oedema were usually severe and the acuity $6 / 30(20 / 100)$ or worse. In a few exceptional cases the leakage was mild, and the initial vision for a short period was surprisingly good $(6 / 9$ to $6 / 18)$ in spite of the broken arcade.

Macular oedema which was observed on the initial angiogram usually resolved during the course of the CRVO, often quickly if the ischaemia was mild and the leakage restricted to the capillaries and venules. If the perifoveal capillary arcade was intact, $84 \%$ of patients achieved a final acuity of $6 / 7.5(20 / 30)$ or better. If, however, the initial angiogram showed the perifoveal net to be broken or absent, $87 \%$ of these patients had a final acuity of $6 / 60(20 / 200)$ or worse, whether or not the macular oedema resolved.

From all the data gathered the linear discriminant analysis showed that, of all variables studied (except A-V transit time, which had incomplete data), those listed in Table 4 were best in predicting the development of rubeosis. For each patient the discriminant score was calculated according to the equation given in Materials and Methods; for example, for patient 13:

$$
\begin{aligned}
& \mathrm{X}=+0 \cdot 4423+(0 \cdot 4128)(1)+(-0 \cdot 1072)(10)+ \\
& (-0 \cdot 1041)(2)+(-0.2265)(3)+0.2870(3)= \\
& -1 \cdot 7630 .
\end{aligned}
$$

A resultant negative value was correlated with a tendency to develop rubeosis; the greater the negative result, the stronger the tendency. The average discriminant score for the rubeosis patients was $-1 \cdot 58$, and for those without rubeosis $+0 \cdot 41$. Discriminant scores of 0 to +2.0 were correlated with probabilities of 0.98 to 1.0 of not developing rubeosis; scores of 0 to -3.00 were correlated with probabilities of 0.56 to 1.00 of developing rubeosis. By means of the discriminant scores for each patient and these probabilities the outcome for 52 of the 57 patients $(91 \%)$ was correctly predicted by the discriminant function.

\section{Discussion}

The incidence of rubeosis iridis and neovascular glaucoma associated with CRVO in this study $(21 \%)$ is in agreement with previous reports (Ashton, 1961; Smith, 1961; Kohner, 1976; Kohner et al., 1976; Laatikainen and Kohner, 1976; Laatikainen and Blach, 1977). The results show that the complication of rubeosis iridis and neovascular glaucoma following CRVO can be predicted with a high degree of accuracy from features of the initial clinical examination and, more importantly, from

Table 4 Discriminant coefficients of variables that best predict rubeosis following CRVO

\begin{tabular}{ll}
\hline Variable & Coefficient \\
\hline Constant $=+0.4423$ & \\
Systemic disease & +0.4128 \\
No. of cotton-wool spots & -0.1072 \\
Macular nonperfusion & -0.8641 \\
Posterior pole nonperfusion & -0.2265 \\
Posterior pole small-vessel permeability & +0.2870 \\
\hline
\end{tabular}

CRVO = central retinal vein occlusion. 
findings of the initial fluorescein angiogram. These variables, summarised in Tables 5 and 6 , are direct or indirect manifestations of an ischaemic infarct and support the concept that anterior segment neovascularisation is correlated with the degree or the extent of fundus ischaemia, or both. The strongest one-to-one correlation was with widespread capillary occlusion as seen on the fluorescein angiogram. Rubeosis iridis was correlated also with the funduscopic observation of cotton-wool spots, but less well than with the extent of capillary occlusion. This was probably because cotton-wool

Table 5 Factors associated with development of rubeosis iridis following $C R V O$

\begin{tabular}{ll}
\hline $\begin{array}{l}\text { Clinical features } \\
\text { Fluorescein angiogram }\end{array}$ & $\begin{array}{l}\text { Patients developing } \\
R I, \%\end{array}$ \\
$\begin{array}{l}\text { Posterior pole or peripheral severe } \\
\text { (3-4 quadrant) capillary occlusion }\end{array}$ & 80 \\
$\begin{array}{l}\text { A-V transit time }>20 \text { s } \\
\begin{array}{l}\text { Posterior pole or peripheral severe large } \\
\text { vessel leakage }\end{array}\end{array}$ & 75 \\
$\begin{array}{l}\text { Posterior pole or peripheral severe small } \\
\text { vessel leakage (excluding disc) }\end{array}$ & 60 \\
$\begin{array}{l}\text { Funduscopy of involved eye } \\
\text { 10 cotton-wool spots }\end{array}$ & 30 \\
$\begin{array}{l}\text { Posterior pole or peripheral severe } \\
\text { retinal oedema }\end{array}$ & 75 \\
$\begin{array}{l}\text { Visual acuity <6/60 } \\
\text { PR }\end{array}$ & $38-57$ \\
\hline
\end{tabular}

$\mathrm{CRVO}=$ central retinal vein occlusion. $\mathrm{RI}=$ rubeosis iridis.

Table 6 Factors that predict no development of rubeosis iridis following CRVO

\begin{tabular}{ll}
\hline Clinical features & $\begin{array}{l}\text { Patients developing } \\
R I, \%\end{array}$ \\
\hline Fluorescein angiogram & $3-5$ \\
No significant areas of capillary occlusion & $4-6$ \\
Minimal large or small vessel leakage & 10 \\
A-V transit time $<20$ s & \\
Funduscopy of involved eye & $7-10$ \\
No retinal oedema (except disc) & 14 \\
No cotton-wool infarcts & 8 \\
Clinical presentation & 7 \\
No evidence of systemic disease & \\
Visual acuity $>6 / 30$ &
\end{tabular}

CRVO = central retinal vein occlusion. ${ }^{\prime} \mathbf{R I}=$ rubeosis iridis. spots became individually less distinguishable as they became more numerous and confluent. Vascular permeability changes, as manifestations of ischaemic injury, showed less significant correlation with the development of rubeosis. This result might be secondary to the difficulty in localising and quantifying the leakage, but it is also possible that factors other than ischaemic damage to the endothelial wall may affect leakage and may not be related to retinal tissue hypoxia.

Prolongation of the A-V transit time was correlated with the development of rubeosis, as observed by Laatikainen and Kohner (1976), but less well than the finding of capillary closure. This observation may be accounted for by the following: blood flow in the large vessels may be partially restored through recanalisation or the growth of collaterals, whereas capillary occlusion continues from ischaemic endothelial cell or pericyte swelling, or perhaps from pressure due to tissue turgor. Hence a prolonged A-V transit time may indicate only indirectly the severity of the ischaemic lesion.

We found that the appearance of disc collateral vessels was not necessarily correlated with the development of rubeosis. These vessels may develop in both ischaemic and non-ischaemic fundi. Even if established, these collaterals may not be adequate to improve the circulation significantly and affect tissue hypoxia. We observed, as did Hayreh (1976), that the fluorescein filling of large retinal vessels was often extremely slowed, in spite of established disc collateral vessels.

Areas of dilated capillaries are observed in fluorescein angiograms as a result of venous obstruction. However, the extent of dilated capillaries was not related to the development of rubeosis iridis in these patients. The area of dilated capillaries was much reduced in eyes with CRVO and severe retinal ischaemia, because of capillary closure over widespread areas.

Visual acuity was a poor predictor of the development of rubeosis iridis, because in the early course of CRVO, as reported also by Laatikainen and Kohner (1976) and Laatikainen (1977a, b), vision was decreased owing to macular oedema as well as perifoveal capillary occlusion (ischaemia). We observed also that perifoveal capillary ischaemia did not necessarily accompany posterior pole or peripheral retinal capillary ischaemia. Occasionally we examined angiograms of patients who had an intact perifoveal capillary arcade with fairly extensive peripheral capillary occlusion. However, the reverse was more often the case: frequently the macula was observed to have excessive capillary loss while the rest of the fundus was minimally affected. This predilection or peculiar sensitivity of 
the posterior pole to ischaemic injury has been noted by Hayreh (1976) and may perhaps be explained by the higher density of photoreceptors and neural elements in the posterior fundus, which cause this area to have higher oxygen requirements, or perhaps by different haemodynamics within different segments of the fundus.

In this study a CRVO associated with systemic, orbital, or ocular disease was correlated with a significantly increased risk of developing anterior segment neovascularisation. However, none of the associated diseases alone showed a statistically significant difference from the cases of rubeosis with no associated disease. This seems to be contrary to the impression of others that rubeosis occurs more frequently in CRVO patients with primary glaucoma or systemic disease (Vannas, 1961; Kottow et al., 1976). Our observation that age was not significantly correlated with rubeosis iridis is also contrary to findings by Vannas (1961) and Kottow et al., (1976), but the increased number of elderly patients having rubeosis with CRVO in this study merely reflected the increased incidence of CRVO in the elderly. This result, however, could be caused by an unrepresentative sample composed of the more severe CRVO patients referred to the Fluorescein Service.

In a previous study of CRVO by Laatikainen and Kohner (1976) it was suggested that a fluorescein angiogram taken 3 months after the occlusion was more accurate in evaluating perifoveal capillary net integrity than the initial angiogram or one taken within 1 month of the onset of symptoms. This was because of progressive deterioration (increased area of capillary occlusion) noted in follow-up angiograms of a few patients. Progression of the ischaemia, if common in CRVO cases, would prohibit early prognostication for rubeosis iridis. To evaluate this we reviewed follow-up fluorescein angiograms taken within 2 to 5 months in 15 patients in whom the initial angiogram was taken within 1 month of the onset of symptoms. The follow-up angiogram in 12 patients did not change significantly; the remaining $3(20 \%)$ showed significant progression or worsening of capillary occlusion, dilatation, and leakage. In 1 of these patients, who had no ischaemia initially, we found scattered foci of capillary occlusion over less than 1 total quadrant. In the second patient, with no initial ischaemia, moderately severe 3-quadrant ischaemia was seen. The third patient, with patchy capillary occlusion, later showed severe confluent occlusion. However, rubeosis iridis developed in none of these 3 . There was no particular feature of the initial history, ocular examination, or fluorescein angiogram that distinguished these patients from those who showed no progressive deterioration.
These results suggest that progressive ischaemic deterioration may occur in CRVO, but that the initial fluorescein angiogram and examination are accurate for most cases in prognosticating the complication of anterior segment neovascularisation. If, however, clinical findings suggest a progression of ischaemia, then perhaps angiography should be repeated. From reviewing these 15 angiograms we agree, however, with Laatikainen and Kohner (1976) that, for the purpose of prognosticating the final visual acuity in CRVO, the 3-month angiogram may be more accurate than the initial angiogram taken within 1 month of the onset of symptoms.

In conclusion, this study has shown that certain features of the initial clinical examination and, more particularly, of the initial fluorescein angiogram which indicate severe retinal ischaemia can prognosticate the development of anterior segment neovascularisation after CRVO. If capillary occlusion over 3 to 4 quadrants is recognised on the fluorescein angiogram, rubeosis iridis will develop in $80-86 \%$ of these patients, but rubeosis will develop in only $3-9 \%$ if minimal occlusion is observed. These extremes, however, represent only a small segment of all CRVO cases. If a discriminant score is calculated utilising capillary occlusion in combination with the other variables that also indicate retinal ischaemia, then up to $91 \%$ of all CRVOs might be accurately prognosticated.

Herbert Kayne performed statistical analysis and S. Flavia Blackwell provided editorial assistance.

Presentation of this paper at ARVO 77 was supported by Fight for Sight, Inc., New York, through a grant from House of Vision, Inc.

\section{References}

Ashton, N. (1961). Neovascularisation in ocular disease. Transactions of the Ophthalmological Societies of the United Kingdom, 81, 145-161.

Hayreh, S. S. (1976). So-called central retinal vein occlusion. Ophthalmologica, 172, 1-37.

Kohner, E. M. (1976). Vision and Circulation, pp. 246-250. C. V. Mosby: St. Louis.

Kohner, E. M., Shilling, J. S., and Hamilton, A. M. (1976). The role of avascular retina in new vessel formation. Metabolic Ophthalmology, 1, 15-23.

Kottow, M., Metzler, U., and Hendrickson, P. (1976). Vision and Circulation, pp. 251-264. C. V. Mosby: St. Louis.

Laatikainen, L. (1977a). Photocoagulation in retinal venous occlusion. Acta Ophthalmologica, 55, 478-488.

Laatikainen, L. (1977b). Preliminary report on effect of retinal panphotocoagulation on rubeosis iridis and neovascular glaucoma. British Journal of Ophthalmology, 61, 278-284.

Laatikainen, L., and Blach, R. K. (1977). Behaviour of the iris vasculature in central retinal vein occlusion: a fluorescein angiographic study of the vascular response of the 
retina and the iris. British Journal of Ophthalmology, 61, 272-277.

Laatikainen, L., and Kohner, E. M. (1976). Fluorescein angiography and its prognostic significance in central retinal vein occlusion. British Journal of Ophthalmology, 60, 411-418.

May, D. R., Klein, M. L., and Peyman, G. A. (1976). A prospective study of xenon arc photocoagulation for central retinal vein occlusion. British Journal of Ophthalmology, 60, 816-818.
Sedney, S. C. (1976). Photocoagulation in retinal vein occlusion. Documenta Ophthalmologica, 40, 1-241.

Shilling, J. S., and Kohner, E. M. (1976). New vessel formation in retinal branch vein occlusion. British Journal of Ophthalmology, 60, 810-815.

Smith, R. (1961). Neovascularisation in ocular disease. Transactions of the Ophthalmological Societies of the United Kingdom, 81, 125-144.

Vannas, S. (1961). Glaucoma due to thrombosis of the central vein of the retina. Ophthalmologica, 142, 266-282. 